\title{
Single-Agent Pixantrone as a Bridge to Autologous Stem Cell Transplantation in a Patient with Refractory Diffuse Large B-Cell Lymphoma
}

\author{
Lorena Appio $^{a} \quad$ Carlo Landoni $^{a} \quad$ Maria La Targia ${ }^{a}$ Vanda Bertollia \\ Martina Chiarucci $^{a}$ Giovanni Crovetti $^{b} \quad$ Elisabetta Vassenna ${ }^{c}$ Giovanni Serio $^{d}$ \\ Marco Bregni ${ }^{a}$

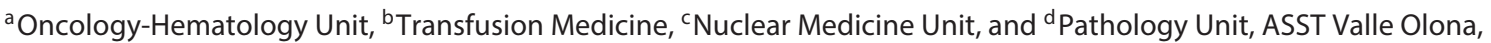 \\ Busto Arsizio, Italy
}

\section{Established Facts}

- Relapsed/refractory aggressive non-Hodgkin lymphoma is associated with poor long-term survival.

- Patients with relapsed non-Hodgkin lymphoma typically receive intensive regimens such as R-ICE (rituximab, ifosfamide, carboplatin, and etoposide) or R-DHAP (rituximab, dexamethasone, cytarabine, and cisplatin), followed by autologous stem cell transplantation for those who respond to chemotherapy.

- Patients who do not achieve a complete response (approx. 50\% of relapsed patients) or who are not candidates for intensive salvage regimens have a poor prognosis, with expected survival of less than 1 year.

- There is no established third-line regimen for patients with aggressive non-Hodgkin lymphoma, and it is very unusual to achieve complete response in third-line treatment.

\section{Novel Insights}

- Pixantrone dimaleate (pixantrone) is a novel aza-anthracenedione, with reduced anthracycline-related cardiotoxicity and comparable antitumour efficacy.

- It is approved in the EU for use as monotherapy in adult patients with multiple relapsed or refractory aggressive B-cell non-Hodgkin lymphoma.

- We report a patient that had a complete response in third-line treatment with pixantrone monotherapy plus a single dose of rituximab, and enjoyed a long-term remission after a consolidation with highdose chemotherapy and stem cell autografting.

- These observations support the use of pixantrone as a bridge to autologous transplant in patients with relapsed/resistant aggressive non-Hodgkin lymphoma.

\section{Keywords}

Refractory lymphoma · Pixantrone · Autologous stem cell transplantation

\begin{abstract}
Aggressive non-Hodgkin lymphoma is associated with poor long-term survival after relapse or resistance to chemotherapy. We report a case of aggressive non-Hodgkin lymphoma refractory to first-line R-CHOP (rituximab, cyclophospha-
\end{abstract}

\section{KARGER}

(c) 2017 S. Karger AG, Basel

E-Mail karger@karger.com

www.karger.com/che
Marco Bregni, MD

Oncology-Hematology Unit, ASST Valle Olona

Ospedale di Circolo di Busto Arsizio, Via Arnaldo Da Brescia 1

IT-21052 Busto Arsizio (IT)

E-Mail marco.bregni@asst-valleolona.it 
mide, doxorubicin, vincristine, and prednisone) and secondline R-DHAP (rituximab, dexamethasone, cytarabine, and cisplatin) chemotherapy treatments. The patient achieved remission with single-agent pixantrone, and received a consolidation with high-dose BEAM (BCNU, etoposide, cytarabine, and melphalan) chemotherapy and autologous stem cell transplantation. He received consolidation radiotherapy on the site of bulky disease. At 20 months from transplant, the disease is in continuous complete remission. The successful use of pixantrone as a bridge to transplant is highlighted, together with the absence of serious side effects.

(c) 2017 S. Karger AG, Basel

\section{Introduction}

Aggressive non-Hodgkin lymphoma is associated with poor long-term survival after relapse, and treatment is limited by a lack of consensus regarding standard of care [1]. The current first-line treatment approach of diffuse large B-cell non-Hodgkin lymphoma (DLBCL) relies to a large extent on anthracycline-based regimens plus anti-CD20 antibody, yielding cure rates in $65-70 \%$ of patients [2]. Despite being highly effective, these regimens are associated with significant long-term toxicity, with a cumulative 10-year incidence of cardiovascular disease of $22 \%$ [3]. Moreover, for the $20-25 \%$ of patients who fail first-line therapy there has been very little progress in terms of salvage regimens over the past 15 years. Retreatment with anthracycline-based regimens was, until now, not an option given the extensive cardiac toxicity of these agents [4]. Patients with relapsed non-Hodgkin lymphoma typically receive intensive regimens such as R-ICE (rituximab, ifosfamide, carboplatin, and etoposide) or RDHAP (rituximab, dexamethasone, cytarabine, and cisplatin), followed by autologous stem cell transplantation (SCT) for those who respond to chemotherapy [5]. For patients who have an inadequate response to, or who are not candidates for intensive salvage regimens, prognosis is poor, with expected survival of less than 1 year. Among patients who are not candidates for SCT, or who relapse following second-line regimens, response rates are low, complete remissions are rare, and expected survival is less than 6 months. There is currently no standard treatment for patients with relapsed/refractory aggressive NHL beyond second-line treatment regimens [6].

Pixantrone dimaleate (pixantrone), a novel aza-anthracenedione, was initially synthesized to reduce anthracycline-related cardiotoxicity without compromising antitumour efficacy. However, pixantrone shows pharmaco-

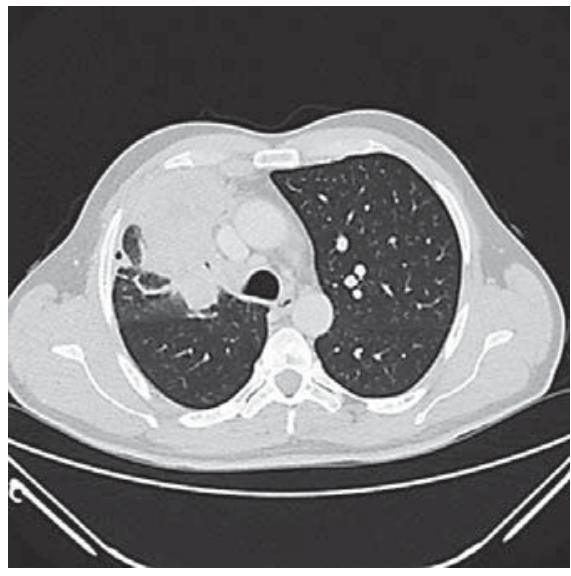

Fig. 1. Chest CT scan at presentation of the disease.

logical and toxicological mechanisms of action that are difficult to reconcile with anthracycline-like drugs, and it is better featured as a new drug with its own characteristics [7]. Unlike anthracyclines and mitoxantrone, pixantrone is only a weak inhibitor of $a$-topoisomerase II. Pixantrone directly alkylates DNA-forming stable DNA adducts and long-lived crosslinks. Furthermore, pixantrone has less potential for generating reactive oxygen species, binding iron, and forming alcohol metabolites that are believed to cause the cardiac toxicity of anthracyclines [8]. According to most recent reports, pixantrone induces a latent type of DNA damage that impairs the fidelity of mitosis, without triggering anthracycline-like DNA damage, but is lethal after successive rounds of aberrant division [9]. In the randomized, open-label, multinational, phase 3 PIX301 trial [10] in patients with multiple relapsed or refractory aggressive non-Hodgkin lymphoma, the complete response plus unconfirmed complete response rate at the end of treatment (primary end point) was significantly higher with intravenous pixantrone monotherapy than with a single-agent comparator (vinorelbine, oxaliplatin, ifosfamide, etoposide, mitoxatrone, or gemcitabine). Progression-free survival was significantly longer in the pixantrone group. Pixantrone was generally well tolerated in PIX301, with a manageable adverse event profile. It is approved in the EU for use as monotherapy in adult patients with multiple relapsed or refractory aggressive B-cell nonHodgkin lymphoma [8]. However, evidence of a longterm benefit from pixantrone is still awaited.

Here we report the case of a heavily pretreated patient with a refractory, aggressive DLBCL who had a favourable long-term outcome after third-line treatment with pixantrone. 

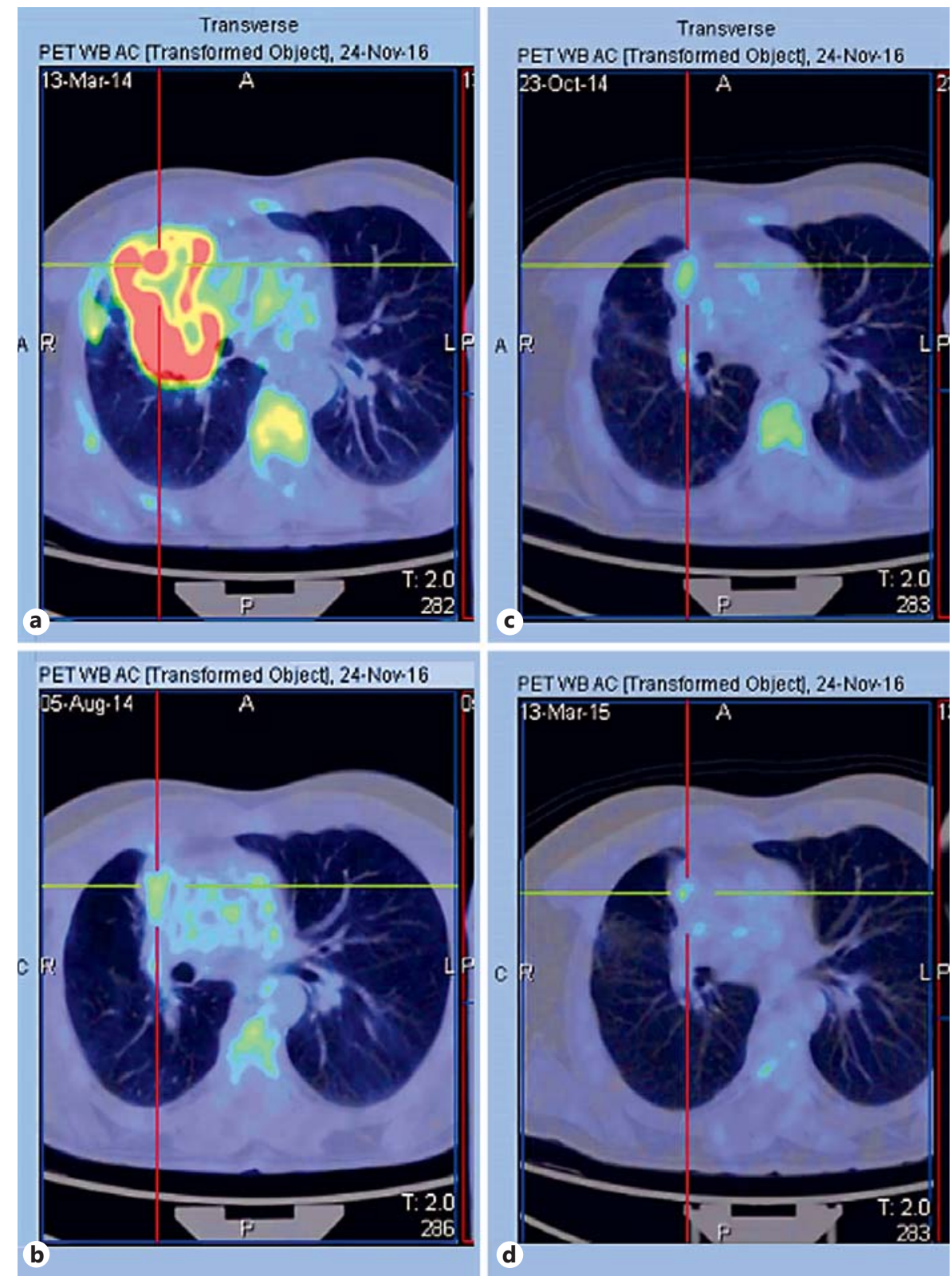

Fig. 2. FDG-PET scan imaging at different times during the course of treatment. a Presentation. b After R-CHOP. c After R-DHAP/R-DHAOx. d After pixantrone.

\section{Case Report}

A 56-year-old man was admitted to our department because of the finding in February 2014 of a voluminous mediastinal lesion involving upper and middle lung lobes (Fig. 1). The patient had experienced B symptoms (low-grade fever, night sweats, and generalized itching) for a few months; he had a history of bronchial asthma. A biopsy of pulmonary nodules was diagnostic for diffuse large B-cell lymphoma CD20+, CD30+, Bcl6+, MUM1+, cyclinD1+/-, Ki67 75\%, ALK1-, HHV8-, and CKMNF116-. An FDG-PET/CT scan confirmed lymph node uptake above and below the diaphragm and the uptake of known pulmonary lesions (Fig. 2).

The bone marrow biopsy was negative. The final diagnosis was DLBCL stage IVB (lung), IPI low, R-IPI good. The patient received first-line immuno-chemotherapy with R-CHOP (rituximab, cyclophosphamide, doxorubicin, vincristine, and prednisone) for 6 courses and obtained a partial remission, so the treatment was followed, according to our standard procedures, by a second-line immuno-chemotherapy with R-DHAP (rituximab, dexamethasone, high-dose cytarabine, and cisplatin) for the first course, and then oxaliplatin was substituted for cisplatin (R-DHAOx) because of the development of a mild renal injury, for a total of 3 courses. After the first cycle, $5 \times 10 \mathrm{e} 6 / \mathrm{kg}$ CD34+ cells were harvested from peripheral blood and cryopreserved. The program was intensification with autologous SCT in the case of a positive response to chemotherapy. However, an FDG-PET/CT scan showed progressive disease at the mediastinal lesion, according to the Lugano classification [11] (Fig. 2). 
The patient then underwent a third-line chemotherapy with pixantrone (Pixuvri ${ }^{\circledR}$ ) $50 \mathrm{mg} / \mathrm{m}^{2}$ on days 1,8 , and 15 every 28 days for 4 courses, plus a single administration of rituximab $375 \mathrm{mg} / \mathrm{m}^{2}$ on day 8 of the third course. The therapy was well tolerated, adverse event was neutropenia, but no infectious complications occurred. FDG-PET/CT demonstrated an overall response with the persistence of a minimal uptake (SUV value 2.92) in the prevascular mediastinum (Fig. 2). In March 2015 the patient underwent high-dose BEAM (BCNU, etoposide, cytarabine, and melphalan) chemotherapy followed by autologous SCT. The treatment was followed by involved-field consolidation radiotherapy on the mediastinum (30 Gy).

A total body CT scan 3 months later showed a complete remission, and also the FDG-PET/CT scan at the last follow-up of August 2016 was completely negative. Left ventricular ejection fraction was $60 \%$ at the presentation, and 60\% in March 2015, after pixantrone and before BEAM. A cardiac reassessment in November 2016, more than 1 year after the completion of radiotherapy, demonstrated a normal cardiac function with $60 \%$ left ventricular ejection fraction.

\section{Discussion}

Autologous SCT remains the only treatment that has demonstrated efficacy for long-term survival in refractory DLBCL [12]. Accordingly, the value of a salvage treatment in multiple relapsed/refractory non-Hodgkin lymphoma patients is to obtain a clinical response (complete response-unconfirmed response) to lead the patient, if eligible, to a consolidation SCT [13]. Several novel agents are undergoing evaluation, but few treatment options are available for this patient population after the failure of established second-line treatments (R-DHAP or R-ICE) [5]. Promising results have been reported with the use of lenalidomide [14] or lenalidomide plus rituximab [15]. In patients who are old and with comorbidities, who are not candidates for SCT, gemcitabine and oxaliplatin have shown efficacy with a good tolerability profile [16]. Pixantrone, given as a single-agent salvage therapy in heavily pretreated patients with relapsed or re- fractory aggressive non-Hodgkin lymphoma, is efficacious and tolerable [12].

Here we report a DLBCL patient whose disease was refractory to 2 lines of treatment and who had a durable remission after treatment with pixantrone and sequential consolidation with autologous SCT. Two notable features of this case are the reversal of chemoresistance with pixantrone and the feasibility of consolidation with SCT. Pixantrone was effective in inducing remission in a tumour that was refractory to multiple agents (anthracyclines, alkylators, antimetabolites); this non-cross-resistance effect is presumably due to its novel mechanism of action, which does not overlap with that of anthracyclines and anthracenediones, and implies interaction with DNA [see ref. 7 for a detailed discussion of the mechanism of action]. The induction of remission achieved with pixantrone allowed the use of SCT as consolidation treatment. The almost 2-year follow-up in continuous complete remission after SCT is predictive of a long-term survival.

In addition, pixantrone was well tolerated without serious side effects. The cardiac performance was not affected by the sequential treatment with anthracyclines and pixantrone in this case.

In conclusion, pixantrone can be a valuable treatment option for patients whose aggressive non-Hodgkin lymphoma has failed to respond to at least 2 previous chemotherapy regimens. Moreover, pixantrone has a favourable adverse event profile. Further results examining the use of pixantrone in combination with rituximab [17] in patients previously treated with rituximab-containing regimens are awaited with interest.

\section{Disclosure Statement}

Marco Bregni participated as a member of a pixantrone advisory board in October 2016. The other authors declare no financial or other potential conflicts of interest.

\section{References}

1 Muszbek N, Kadambi A, Lanitis T, et al: The cost-effectiveness of pixantrone for third/ fourth-line treatment of aggressive nonHodgkin's lymphoma. Clin Ther 2016;38: 503-515.

2 National Comprehensive Cancer Network guidelines, version 2.2016. Diffuse large B-cell lymphoma. NCCN, 2016.

3 Moser EC, Noordijk EM, von Leeuwen FE, et al: Long-term risk cardiovascular disease after treatment for aggressive non-Hodgkin lymphoma. Blood 2006;107:2912-2919.
4 Swain S, Whaley FS, Ewe MS: Congestive heart failure in patients treated with doxorubicin: a retrospective analysis of three trials. Cancer 2003;97:2869-2879.

5 Gisselbrecht C, Glass B, Mounier N, et al: Salvage regimens with autologous transplantation for relapsed large B-cell lymphoma in the rituximab era. J Clin Oncol 2010;28:41844190 
6 Chao MP: Treatment challenges in the management of relapsed or refractory non-Hodgkin's lymphoma - novel and emerging therapies. Cancer Manag Res 2013;5:251-269.

7 Menna P, Salvatorelli E, Minotti G: Rethinking drugs from chemistry to therapeutic opportunities: pixantrone beyond anthracyclines. Chem Res Toxicol 2016;29:1270-1278.

8 Pean E, Flores B, Hudson I, et al: The European Medicines Agency review of pixantrone for the treatment of adult patients with multiple relapsed or refractory aggressive nonHodgkin's B-cell lymphomas: summary of the scientific assessment of the committee for medicinal products for human use. Oncologist 2013;18:625-633.

9 Beeharry N, Ghelli Luserna Di Rora A, Smith $M R$, et al: Pixantrone induces cell death through mitotic perturbations and subsequent aberrant cell divisions. Cancer Biol Ther 2015;16:1397-1406.
10 Pettengell R, Coiffier B, Narayanan G, et al: Pixantrone dimaleate versus other chemotherapeutic agents as a single-agent salvage treatment in patients with relapsed or refractory aggressive non-Hodgkin lymphoma: a phase 3, multicentre, open-label, randomized trial. Lancet 2012;13:696-706.

11 Cheson BD, Fisher RI, Barrington SF, et al: Recommendations for initial evaluation, staging, and response assessment of Hodgkin and non-Hodgkin lymphoma: the Lugano classification. J Clin Oncol 2014;32:3059-3067.

12 Martelli M, Ferreri AJM, Agostinelli C, et al: Diffuse large B-cell lymphoma. Crit Rev Oncol Hematol 2013;87:146-171.

13 Zinzani PL, Corradini P, Martelli M, et al: Critical concepts, practice recommendations, and research perspectives of pixantrone therapy in non-Hodgkin lymphoma: a SIE, SIES, and GITMO consensus paper. Eur J Haematol 2015;97:554-561.
14 Mondello P, Steiner N, Willenbacher W, et al: Lenalidomide in relapsed or refractory diffuse large B-cell lymphoma: is it a valid treatment option? Oncologist 2016;21:1107-1112.

15 Zinzani PL, Pellegrini C, Argnani L, et al: Prolonged disease-free survival in elderly relapsed diffuse large B-cell lymphoma patients treated with lenalidomide plus rituximab. Haematologica 2016;101:e385-e386.

16 El Gnaoui T, Dupuis J, Belhadj K, et al: Rituximab, gemcitabine and oxaliplatin: an effective salvage regimen for patients with relapsed or refractory B-cell lymphoma not candidates for high-dose therapy. Ann Oncol 2007;18: 1363-1368.

17 Belada D, Georgiev P, Dakhil S, et al: Pixantrone-rituximab versus gemcitabine-rituximab in relapsed/refractory aggressive nonHodgkin lymphoma. Future Oncol 2016;12: 1759-1768. 